\title{
Abnormal Structure of Fear Circuitry in Pediatric Post-Traumatic Stress Disorder
}

\author{
Taylor J Keding' and Ryan J Herringa*,' \\ 'Department of Psychiatry, University of Wisconsin School of Medicine and Public Health, Madison, WI, USA
}

\begin{abstract}
Structural brain studies of adult post-traumatic stress disorder (PTSD) show reduced gray matter volume (GMV) in fear regulatory areas including the ventromedial prefrontal cortex (VmPFC) and hippocampus. Surprisingly, neither finding has been reported in pediatric PTSD. One possibility is that they represent age-dependent effects that are not fully apparent until adulthood. In addition, lowerresolution MRI and image processing in prior studies may have limited detection of such differences. Here we examine fear circuitry GMV, including age-related differences, using higher-resolution MRI in pediatric PTSD vs healthy youth. In a cross-sectional design, 3 T anatomical brain MRI was acquired in 27 medication-free youth with PTSD and 27 healthy non-traumatized youth of comparable age, sex, and IQ. Voxel-based morphometry was used to compare GMV in a priori regions including the medial prefrontal cortex and amygdala/hippocampus. Compared with healthy youth, PTSD youth had reduced GMV but no age-related differences in anterior vmPFC (BA IO/II, Z = 4.5), which inversely correlated with PTSD duration. In contrast, although there was no overall group difference in hippocampal volume, a group $\times$ age interaction $(Z=3.6)$ was present in the right anterior hippocampus. Here, age positively predicted hippocampal volume in healthy youth but negatively predicted volume in PTSD youth. Within the PTSD group, re-experiencing symptoms inversely correlated with subgenual anterior cingulate cortex (sgACC, $Z=3.7)$ and right anterior hippocampus $(Z=3.5)$ GMV. Pediatric PTSD is associated with abnormal structure of the vmPFC and age-related differences in the hippocampus, regions important in the extinction and contextual gating of fear. Reduced anterior vmPFC volume may confer impaired recovery from illness, consistent with its role in the allocation of attentional resources. In contrast, individual differences in sgACC volume were associated with re-experiencing symptoms, consistent with the role of the sgACC in fear extinction. The negative relationship between age and hippocampal volume in youth with PTSD may suggest an ongoing neurotoxic process over development, which further contributes to illness expression. Future studies employing a longitudinal design would be merited to further explore these possibilities.

Neuropsychopharmacology (2015) 40, 537-545; doi: 10.1 038/npp.20 I4.239; published online 8 October 2014
\end{abstract}

\section{INTRODUCTION}

Pediatric post-traumatic stress disorder (PTSD) is a debilitating and common illness, affecting five percent of youth by the age of 18 (Merikangas et al, 2010). Pediatric PTSD has high comorbidity with other psychiatric illnesses including anxiety disorders, depression, and ADHD (Famularo et al, 1996). Furthermore, adults with PTSD related to childhood trauma have a higher incidence of depression, suicide attempts, and substance abuse (Warshaw et al, 1993). These findings highlight the need to better understand the underlying pathophysiology of pediatric PTSD, with the aim of mitigating its effects.

PTSD has been characterized clinically and behaviorally by impaired fear extinction (Pitman et al, 2012). Furthermore, neuroimaging studies of adult PTSD suggest structural

\footnotetext{
*Correspondence: Dr RJ Herringa, Department of Psychiatry, University of Wisconsin School of Medicine and Public Health, 6001 Research Park Boulevard, Madison, WI 53719, USA, Tel: +608 263 6068, Fax: +608 262 9246, E-mail: herringa@wisc.edu

Received 20 June 2014; revised 5 September 2014; accepted 7 September 2014; accepted article preview online 12 September 2014
}

abnormalities in fear regulatory areas, namely, smaller gray matter volume (GMV) of the ventromedial prefrontal cortex (vmPFC) including anterior cingulate cortex (ACC), and hippocampus (Pitman et al, 2012). The vmPFC is notable for its role in the extinction of fear responses via inhibition of the amygdala (Milad and Quirk, 2012), whereas the hippocampus can contextually limit fear responses via connections to both the amygdala and vmPFC (Maren et al, 2013). Of note, the amygdala, which frequently shows hyperactivation in adult PTSD (Pitman et al, 2012), has not shown any clear structural abnormalities in meta-analyses (Kühn and Gallinat, 2013; Woon and Hedges, 2009). However, a recent study examining a large cohort did find evidence of smaller amygdala volume in adult PTSD (Morey et al, 2012).

Meta-analyses in adults suggest that both trauma exposure and PTSD are associated with reduced hippocampal volume, with this effect greater in PTSD (Karl et al, 2006; Kühn and Gallinat, 2013; Woon et al, 2010). There remains debate as to whether smaller hippocampal volume represents a premorbid or acquired trait in PTSD. A twin study of adult PTSD suggests the former (Gilbertson et al, 2002), while leaving open the possibility of common developmental 
effects in the PTSD twin pairs. In contrast, reduced vmPFC volume appears to be an acquired trait that contributes to the emergence of PTSD in vulnerable adults (Kasai et al, 2008; Sekiguchi et al, 2013). In further evidence of their role in adult PTSD, both vmPFC (Herringa et al, 2012; Sekiguchi et al, 2013) and hippocampal volume (Apfel et al, 2011; Gilbertson et al, 2002; Karl et al, 2006) inversely correlate with PTSD severity. Successful treatment may reverse hippocampal volume deficits (Levy-Gigi et al, 2013; Vermetten et al, 2003), but does not appear to affect vmPFC volume (Dickie et al, 2013; Levy-Gigi et al, 2013). However, pretreatment vmPFC volume is associated with symptom reduction over time (Bryant et al, 2008; Dickie et al, 2013).

Surprisingly, structural brain studies of pediatric PTSD have so far not found any reduction in hippocampal or vmPFC GMV compared with healthy youth. In contrast to adult PTSD, a meta-analysis did not find hippocampal volume differences in pediatric PTSD, but did find reduced volume in child abuse-related adult PTSD, suggesting a delayed developmental effect (Woon and Hedges, 2008). In partial support of this, a pilot study of 15 youth with post-traumatic stress symptoms (PTSS) found an inverse correlation between baseline PTSS and cortisol and hippocampal volume change over 12-18 months (Carrion et al, 2007). However, the absence of a control group limits interpretation of these findings. Prefrontal GMV findings in pediatric PTSD have been mixed. Findings include no difference in total prefrontal GMV in pediatric PTSD (De Bellis et al, 1999, 2002; De Bellis and Keshavan, 2003), and greater vmPFC GMV in youth with PTSS (Carrion et al, 2009; Richert et al, 2006). In addition, prior studies have not found any relationship between total prefrontal GMV (De Bellis et al, 1999, 2002; De Bellis and Keshavan, 2003; Carrion et al, 2001) or PFC subdivisions (Richert et al, 2006) and pediatric PTSD severity. Finally, a meta-analysis did not find any differences in amygdala volume in pediatric PTSD (Woon and Hedges, 2008).

Delayed developmental effects offer one explanation for the discrepancy in structural brain findings between pediatric and adult PTSD. In addition, lower-resolution MRI and processing techniques in prior structural brain studies of pediatric PTSD, although standard at the time, may have limited the ability to detect localized differences in GMV. These findings highlight the need for a new assessment of structural brain differences in pediatric PTSD using current methodologies, while also taking into account potential age-related effects.

To this end, we report the results of structural brain MRI analysis in pediatric PTSD compared with non-traumatized healthy youth using a cross-sectional design. We used higher-resolution (3 T) MRI and high-dimensional normalization along with voxel-based morphometry (VBM) to examine brain-wide differences in GMV. We also examined age-related differences in GMV as an indicator of altered gray matter development in pediatric PTSD. We conducted these analyses within a priori regions including the medial prefrontal cortex and amygdala/hippocampus complex, with additional reporting of whole-brain findings. We predicted that youth with PTSD would have reduced vmPFC volume, and that age would negatively predict hippocampal volume. The latter would suggest a process counter to typical hippocampal development, which increases in volume through early adulthood (Uematsu et al, 2012).

Furthermore, we examined the relationship of regional GMV with PTSD symptom cluster severity, with the aim of mapping the neurobiological substrates of specific symptom clusters. We predicted that re-experiencing symptoms in particular would be inversely related to GMV of the vmPFC and hippocampus, given the link between re-experiencing and impaired fear extinction in adult PTSD (Norrholm et al, 2011). Finally, it is important to note that the current study does not contain a trauma-exposed healthy comparison group, which may help delineate more general effects of trauma from PTSD symptoms. To address this, we conducted post hoc analyses examining the relationship of GMV abnormalities with both PTSD and trauma exposure measures.

\section{MATERIALS AND METHODS}

\section{Participants}

Twenty-seven medication-free youth with PTSD and twenty-eight non-traumatized healthy youth between the ages of 8 and 18 years were recruited from local mental health clinics and through local advertisements, respectively. Exclusion criteria for all participants included IQ $<70$, unstable medical condition, MRI contraindication, and possibility of pregnancy in females. Additional exclusion criteria for youth with PTSD included active suicidality, history of psychotic disorder, bipolar disorder, or OCD; recent (past 4 weeks) substance abuse or dependence; and recent use of psychotropic medication (past 4 weeks; 6 weeks for fluoxetine). Participants were not taken off psychotropic medications for the purposes of the study. Healthy control subjects were required to be free of any history of mental illness. All procedures were approved by the University of Wisconsin Health Sciences IRB, and parental consent and youth assent were obtained for all participants.

\section{Clinical Assessment}

All participants underwent a trauma and psychiatric screen by a board-certified child psychiatrist $(\mathrm{RJH})$ with the Kiddie Schedule for Affective Disorders and Schizophrenia (KSADS; Kaufman et al, 1997) incorporating both youth and caregiver reports. A PTSD diagnosis was determined using DSM-IV criteria by combination of the KSADS and Clinician-Administered PTSD Scale for Children and Adolescents (CAPS-CA; Nader et al, 1998; Weathers et al, 2001). A PTSD diagnosis required at least five DSM-IV symptoms, including at least one from each symptom cluster, following Cohen et al (2011). These criteria are slightly modified from adult criteria and were chosen to allow greater likelihood of study inclusion, yet maintain a relatively high overall symptom severity. Furthermore, youth fulfilling two $v s$ three symptom clusters do not differ in overall clinical impairment or distress (Carrion et al, $2002)$. With these modified criteria, most youth $(n=22$ or $82 \%$ ) in the PTSD group met full standard DSM-IV criteria for PTSD. Of the other five PTSD participants, three met criteria for two of three symptom clusters, and two met criteria for one of three symptom clusters. Note that the CAPS-CA was not obtained for the first seven PTSD 
participants. PTSD severity was additionally examined using the UCLA PTSD Reaction Index (PTSD-RI; Steinberg et al, 2004). For the PTSD-RI, the greater of youth and caregiver report for each item was used, as this was most strongly correlated with CAPS scores $(r=0.85,0.74$, and 0.60 for greater of youth/caregiver, youth only, and caregiver only, respectively). Depressive symptoms over the past 2 weeks were quantified with the Mood and Feelings Questionnaire (MFQ; Costello and Angold, 1988). Anxiety symptoms over the past 3 months were quantified with the Screen for Child Anxiety Related Emotional Disorders (SCARED; Birmaher et al, 1997). MFQ and SCARED scores were calculated using the average of youth and caregiver reports. Pubertal stage was assessed by self-report using the Tanner picture-based rating scale (Morris and Udry, 1980). IQ was estimated using the Full-Scale IQ-2 component of the Wechsler Abbreviated Scale of Intelligence-II (Wechsler, 2011). One participant with PTSD was unable to complete IQ testing due to fatigue.

\section{MRI Acquisition}

All youth completed two mock scan sessions before MRI to accommodate them to the scanning environment. Anatomical brain MRI was acquired using a 3.0 T GE Discovery MR750 scanner (General Electric, Milwaukee, WI) at the University of Wisconsin Department of Psychiatry. Threedimensional axial high-resolution T1 images were acquired with the following parameters: TE: $3.18 \mathrm{~ms}$, TR: $8.16 \mathrm{~ms}$, TI: $450 \mathrm{~ms}$, slice thickness: $1 \mathrm{~mm}, 156$ slices, flip angle: $12^{\circ}$, FOV: $25.6 \mathrm{~cm}$, and image matrix $256 \times 256$ that covered the entire brain.

\section{Image Preprocessing and VBM}

Preprocessing and VBM were performed using the VBM8 toolbox (http://dbm.neuro.uni-jena.de/vbm/) in Statistical Parametric Mapping, version 8 (SPM8, Wellcome Department of Imaging Neuroscience, London, UK), which was executed in Matlab R2011b (Mathworks, Sherborn, MA). Standard VBM8 toolbox parameters were used for preprocessing. Images were bias corrected, tissue classified, and normalized to MNI space using linear (12-parameter affine) and nonlinear transformations including highdimensional DARTEL within a unified model. Standard values were selected to bias regularization $(0.0001)$ and FWHM cutoff $(60 \mathrm{~mm})$. Denoising was performed with optimized Rician non-local means and HMRF weighting of 0.15 . Output-normalized GMVs were modulated for nonlinear (Jacobian) components only, resulting in images corrected for total brain volume and smoothed with a 6-mm FWHM Gaussian filter. Final voxel resolution was $1.5 \times$ $1.5 \times 1.5 \mathrm{~mm}$. Gray matter images were checked for homogeneity using the covariance structure of each image with all other images. One control participant was excluded due to covariance greater than two SD below the mean (movement artifact), leaving 27 control youth in the final analysis.

\section{Statistical Analysis}

Processed GMVs were entered into a two-sample $t$-test within SPM8 comparing PTSD with controls while covarying for age and sex. A group $\times$ age interaction term was included to examine age-related differences between the two groups cross-sectionally. Finally, a multivariate regression was performed within the PTSD group only, testing the association of GMV with PTSD-RI symptom cluster scores (re-experiencing, avoidance, and hyperarousal), covaried for age and sex. A priori search regions included the mPFC and amygdala/hippocampus complex, using regional masks created in AFNI (Cox, 1996). The mPFC mask included ventromedial PFC, ACC (including subgenual, rostral, and dorsal regions), and dorsomedial PFC. The more dorsal regions of the mPFC were included in our search region, given evidence that GMV abnormalities may extend into these regions in adult PTSD (Meng et al, 2014). Multiple comparison correction was performed using Monte Carlo simulation (AFNI's 3dClustSim), which incorporates the estimated smoothness of the data to establish the likelihood of false positives of different cluster sizes (that is, cluster size thresholding). At an individual voxel threshold of $P \leqslant 0.001$, the cluster threshold was 139 voxels for the mPFC and 13 voxels for the amygdala/hippocampus, resulting in a corrected $\alpha \leqslant 0.05$. Additional results are reported outside of these regions surviving whole-brain correction (cluster threshold 351 voxels). Secondary analyses of extracted cluster data were performed in SPSS v. 21 (IBM, Armonk, NY) to examine their relationship with PTSD symptoms, illness duration, time elapsed since the index trauma, index trauma type, number of trauma types experienced, as well as potentially confounding variables including IQ, pubertal stage, depressive symptoms, general anxiety symptoms, and past medication exposure.

\section{RESULTS}

\section{Participants}

Participant characteristics are displayed in Table 1. The groups were similar in terms of sex distribution, age, Tanner stage, IQ, and handedness ( $p>0.05, \chi^{2}$ or $t$-test). Within the PTSD group, the most common index trauma was sexual abuse, followed by traumatic death of a loved one, accident, and witnessing violence. PTSD symptoms averaged 47.3 based on the PTSD-RI, which is indicative of severe PTSD. Of the 27 PTSD youth, 24 had comorbid psychiatric illness, most commonly depressive disorders $(n=19)$.

\section{Voxel-Based Morphometry}

VBM results are summarized in Table 2. The group contrast revealed significantly smaller GMV in the right anterior vmPFC (BA 10/11; Figure 1) in PTSD youth. There were no age-related differences in vmPFC GMV between the groups, with age negatively predicting GMV similarly in healthy and PTSD youth (Figure 2). In the hippocampus, there were no overall group differences in GMV, but there was a significant group $\times$ age interaction in the right anterior hippocampus. Here, age positively predicted hippocampal GMV in healthy youth, but negatively predicted GMV in PTSD youth (adjusted $r=0.50$ and -0.48 , respectively; Figure 1). Outside of a priori search regions, PTSD youth had reduced GMV in bilateral fusiform gyrus 
Table I Participant Characteristics

\begin{tabular}{|c|c|c|}
\hline & Control & PTSD \\
\hline N & $27(\mathrm{I} 3 \mathrm{~F})$ & $27(\mathrm{I} 8 \mathrm{~F})$ \\
\hline Age & $13.6( \pm 3.0)$ & $14.2( \pm 2.7)$ \\
\hline Tanner stage & $3.1( \pm 1.4)$ & $3.4(1.2)$ \\
\hline IQ & $108.8( \pm 12.9)$ & $102.2( \pm 12.2)$ \\
\hline Left handed (n) & । & 3 \\
\hline \multirow{4}{*}{ Index trauma $(n)$} & - & Sexual abuse $(10)$ \\
\hline & & Witnessing violence (4) \\
\hline & & Traumatic death of loved one (8) \\
\hline & & Accident (5) \\
\hline \multirow{6}{*}{$\begin{array}{l}\text { Comorbid diagnoses } \\
\text { (n) }\end{array}$} & - & Major depressive disorder (I7) \\
\hline & & $\begin{array}{c}\text { Depressive disorder not otherwise } \\
\text { specified (2) }\end{array}$ \\
\hline & & Separation anxiety disorder (6) \\
\hline & & Social anxiety disorder (3) \\
\hline & & Generalized anxiety disorder (3) \\
\hline & & ADHD (8) \\
\hline PTSD duration & - & $46( \pm 36)$ months \\
\hline PTSD Reaction Index & - & $47.3( \pm 13.5)$ \\
\hline $\begin{array}{l}\text { CAPS-CA past } \\
\text { month }\end{array}$ & - & $67.8( \pm 20.5)$ \\
\hline MFQ & $2.7( \pm 2.1)$ & $24.2( \pm 10.4)$ \\
\hline SCARED & $6.6( \pm 4.5)$ & $31.9( \pm 13.6)$ \\
\hline Past psychiatric & - & Stimulant (8) \\
\hline \multirow[t]{3}{*}{ medication (n) } & & Antidepressant (7) \\
\hline & & Alpha-2 agonist (I) \\
\hline & & Benzodiazepine (I) \\
\hline
\end{tabular}

Abbreviations: CAPS-CA, Clinician-Administered PTSD Scale Child and

Adolescent version; MFQ, Mood and Feelings Questionnaire; SCARED, Screen for Child Anxiety Related Emotional Disorders.

The control and PTSD groups did not significantly differ in sex distribution, age, Tanner stage, IQ, or handedness. The PTSD Reaction Index was determined by the greater of youth and caregiver scores for each item. The MFQ and SCARED represent the average of youth and caregiver reports. Note that the CAPS-CA was not obtained for the first seven PTSD participants. Numbers in parentheses represent SD unless otherwise indicated.

(Supplementary Figure S1; Table 2) and left occipital cortex (BA 18/19, Supplementary Figure S2; Table 2). There was also evidence for a group $\times$ age interaction in the same left occipital cortex area, although this did not survive cluster thresholding $(k=198$; Supplementary Figure S2). No group differences were observed for amygdala GMV, and there were no regions in which PTSD youth had greater GMV than controls.

Within the PTSD group, the multivariate regression of PTSD symptom clusters (covaried for age and sex) revealed an inverse correlation between re-experiencing symptoms and GMV in the right subgenual ACC (sgACC, BA 25) and right anterior hippocampus (adjusted $r=-0.71$ and -0.69 , respectively; Figure 3 ). No significant associations were observed with avoidance/numbing or hyperarousal symptoms. Consistent with the anterior vmPFC cluster derived from the group contrast, a plot of sgACC volume revealed that age negatively predicted GMV similarly in both groups (Figure 2).

\section{Secondary Analyses}

Secondary analyses conducted in SPSS are fully described under Supplementary Information and briefly summarized here. Group contrast and PTSD symptom association results did not markedly change when covaried for IQ, Tanner stage, depressive symptoms, general anxiety symptoms, and past months of exposure to ADHD and antidepressant medication. In addition, no sex interactions were found.

Multivariate regression analyses were conducted within the PTSD group to examine the relationship of each cluster with PTSD and trauma exposure measures. Results are summarized in Table 2. Multiple regions showed relationships with PTSD duration and symptom clusters (but not total PTSD severity), with few associations with trauma exposure measures. These and further analyses are described in Supplementary Information.

\section{DISCUSSION}

To our knowledge, this is the first reported study to examine structural and age-related brain differences in pediatric PTSD using higher-resolution (3 T) MRI and highdimensional normalization with VBM. Our results suggest that pediatric PTSD, compared with non-traumatized healthy youth, is characterized by reduced vmPFC volume and age-related differences in the hippocampus, the latter of which may suggest an abnormal developmental process. In contrast to the hippocampus, we did not find any evidence for age-related differences in the vmPFC. We further found that re-experiencing symptoms are inversely associated with sgACC and hippocampus GMV. Consistent with prior structural brain studies of pediatric PTSD, we did not observe any differences in amygdala GMV. In secondary analyses, vmPFC and hippocampus GMV were associated primarily with clinical measures of PTSD (as opposed to trauma exposure), implicating these regions in the pathophysiology of pediatric PTSD. Outside of our a priori search regions, pediatric PTSD was also characterized by reduced volume in visual processing areas (see Supplementary Information for further discussion).

Reduced vmPFC volume has been a replicated finding in adult PTSD (Pitman et al, 2012) but has so far not been observed in pediatric PTSD. Interestingly, the only prior VBM study of pediatric PTSD suggested increased sgACC volume (Carrion et al, 2009), which was more posterior to our anterior vmPFC cluster but overlapping with our sgACC cluster inversely correlated with re-experiencing symptoms. Interestingly, the prior VBM study also reported increased occipital cortex GMV, which also contrasts with findings in the current study. Aside from methodological differences in MRI acquisition and analysis, PTSD sample characteristics could also account for discrepant findings between these two studies. For example, in the sample of Carrion et al, $50 \%$ of youth met standard criteria for PTSD compared with $82 \%$ in the current sample. One possibility, then, is that 
Table 2 Summary of Voxel-Based Morphometry Results

\begin{tabular}{|c|c|c|c|c|c|c|c|c|c|c|}
\hline Contrast & Region & BA & Cluster voxels & Peak Z & $x$ & $y$ & $\mathbf{z}$ & $\begin{array}{l}\text { Post hoc regression } \\
\text { (PTSD only) }\end{array}$ & Std. beta & $p$-value \\
\hline \multirow[t]{3}{*}{ Control > PTSD } & R. ant. vmPFC & 10,11 & 504 & 4.47 & 16 & 62 & -2 & $\begin{array}{l}\text { Age } \\
\text { PTSD duration }\end{array}$ & $\begin{array}{l}-0.451 \\
-0.661\end{array}$ & $\begin{array}{l}0.009 \\
0.018\end{array}$ \\
\hline & R. fusiform gyrus & 20,36 & 649 & 4.42 & 52 & -24 & -26 & Avoidance/numbing & 0.522 & 0.090 \\
\hline & L. fusiform gyrus & 20,36 & 449 & 4.25 & -48 & -31 & -20 & - & & \\
\hline Group $\times$ age: control $>$ PTSD & R. ant. hippocampus & - & 94 & 3.58 & 32 & -12 & -17 & $\begin{array}{l}\text { Age } \\
\text { Re-experiencing } \\
\text { Hyperarousal } \\
\text { PTSD duration } \\
\text { Time since trauma }\end{array}$ & $\begin{array}{r}-0.574 \\
-0.364 \\
0.376 \\
-0.543 \\
0.682\end{array}$ & $\begin{array}{l}0.003 \\
0.089 \\
0.074 \\
0.060 \\
0.058\end{array}$ \\
\hline $\begin{array}{l}\text { Cluster B-re-experiencing } \\
\text { symptoms }(-)\end{array}$ & R. ant. hippocampus & - & 51 & 3.51 & 38 & -15 & -27 & $\begin{array}{l}\text { Re-experiencing } \\
\text { Avoidance/numbing } \\
\text { Hyperarousal }\end{array}$ & $\begin{array}{r}-0.701 \\
0.721 \\
0.308\end{array}$ & $\begin{array}{c}<0.001 \\
<0.001 \\
0.043\end{array}$ \\
\hline
\end{tabular}

Abbreviations: sgACC, subgenual anterior cingulate cortex; vmPFC, ventromedial prefrontal cortex.

Clusters shown survived cluster thresholding (corrected $\alpha \leqslant 0.05$ ) within a priori search regions of the medial prefrontal cortex or amygdala/hippocampus complex. Additional clusters outside of these regions surviving whole-brain correction are also displayed. Peak coordinates $(x, y$, and $z)$ are based on the MNI atlas in RAl orientation. All analyses included age and sex as covariates. The association with DSM-IV cluster B symptoms was conducted as part of a multivariate regression, including cluster $C$ and D symptoms of PTSD. Post hoc analysis was performed within the PTSD group on extracted cluster data using multivariate regression to examine relationships with PTSD and trauma exposure measures. The model included age, sex, DSM-IV PTSD B/C/D symptoms, PTSD duration, number of trauma types endorsed, and time elapsed since the initial index trauma. Significant or trend-level findings are displayed.
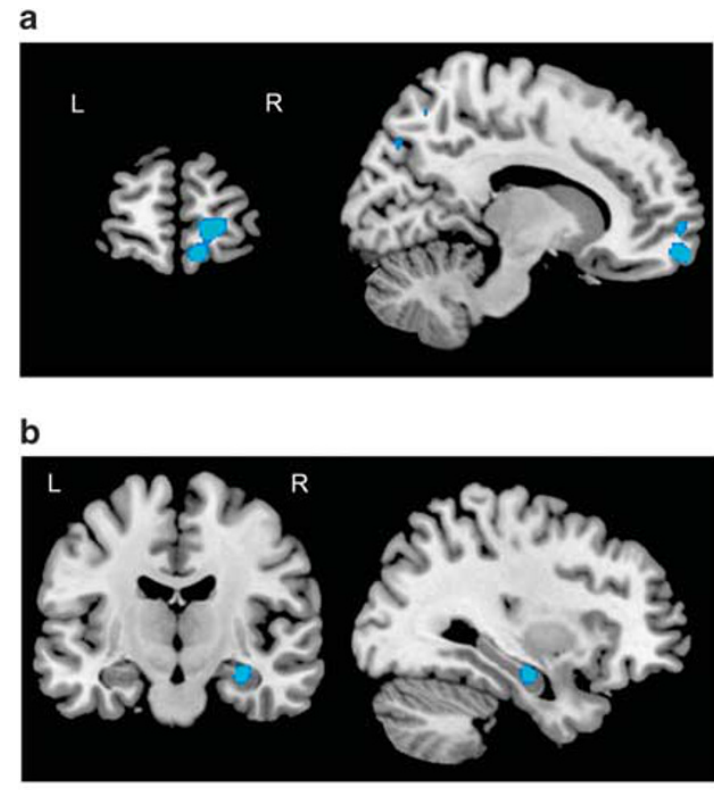
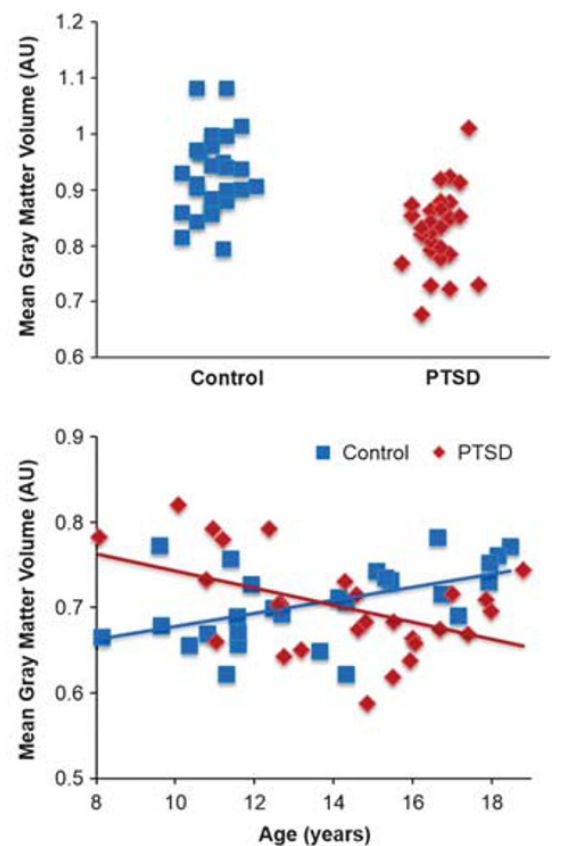

Figure I Gray matter volume (GMV) abnormalities in pediatric PTSD compared with healthy youth. (a) Reduced right anterior vmPFC GMV in youth with PTSD, covaried for age and sex. (b) Age-related differences in right anterior hippocampus in pediatric PTSD compared with healthy youth. A group $\times$ age interaction, covaried for sex, revealed that age positively predicted hippocampal GMV in healthy youth, but negatively predicted GMV in youth with PTSD. Scatterplots of extracted cluster data are shown on the right. PTSD, post-traumatic stress disorder; vmPFC, ventromedial prefrontal cortex. $n=27$ per group. 

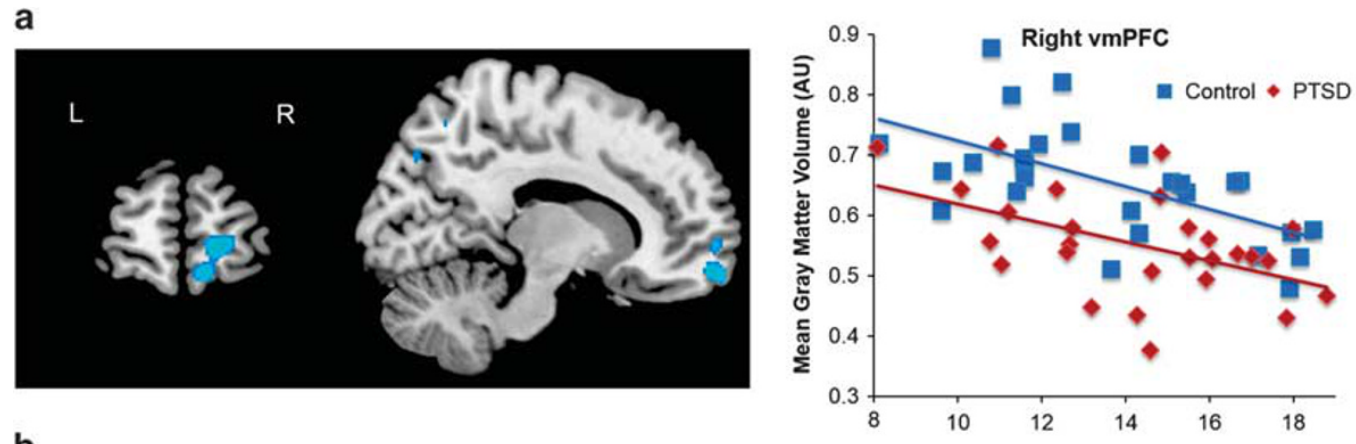

b
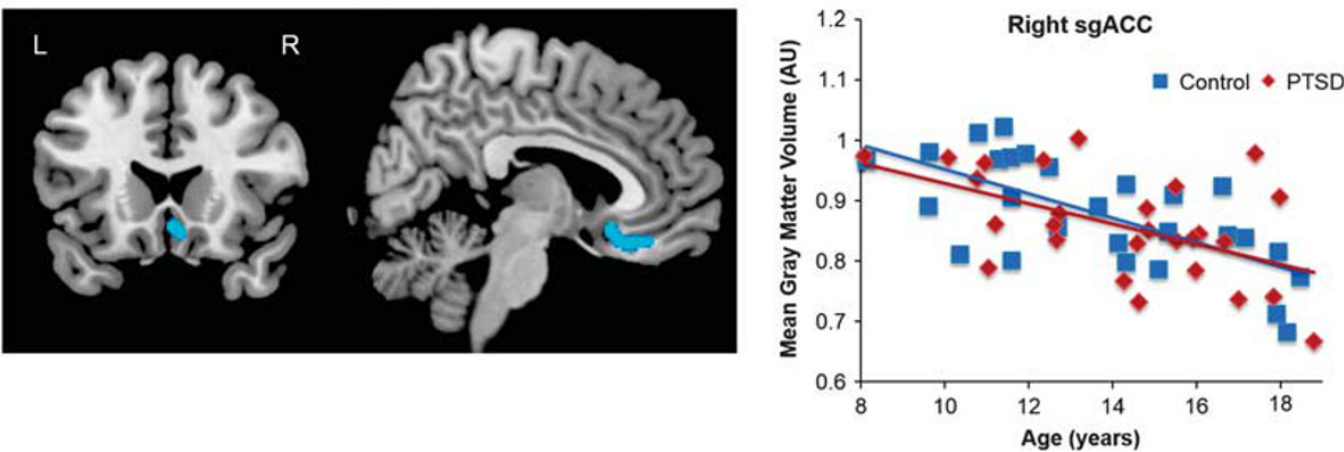

Figure 2 Age-dependent changes in VmPFC gray matter volume (GMV) do not differ between pediatric PTSD and healthy youth. Shown here are (a) right anterior vmPFC that exhibited overall reduced GMV in pediatric PTSD from Figure I and (b) right sgACC that was inversely correlated with re-experiencing symptoms from Figure 3. Scatterplots of extracted cluster data in relation to age are shown on the right. sgACC, subgenual anterior cingulate cortex; vmPFC, ventromedial prefrontal cortex. $n=27$ per group.

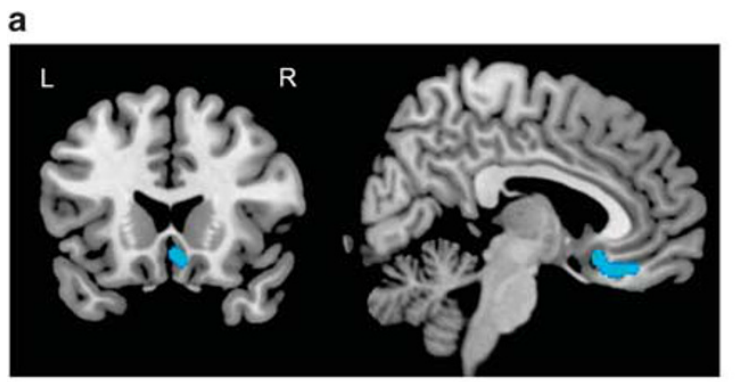

b

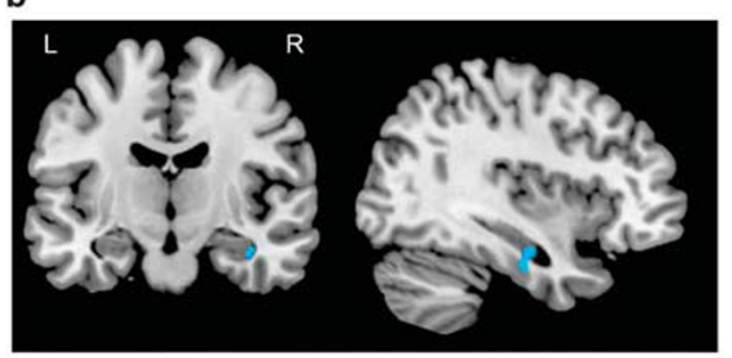

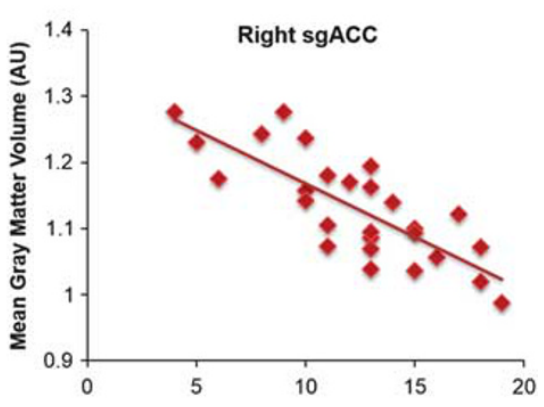

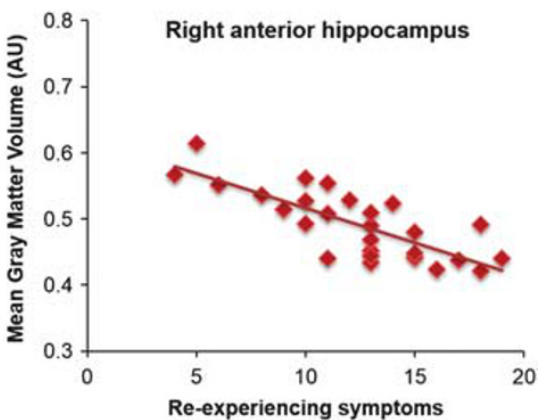

Figure 3 Re-experiencing (DSM-IV cluster B) symptoms of PTSD are inversely correlated with (a) right sgACC and (b) right anterior hippocampus gray matter volume. Scatterplots on the right show extracted cluster data in relation to re-experiencing symptom severity determined by the UCLA PTSD Reaction Index. Results include sex, age, and cluster $C$ and D symptoms as covariates. PTSD, post-traumatic stress disorder; sgACC, subgenual anterior cingulate cortex; vmPFC, ventromedial prefrontal cortex. $n=27$.

reduced vmPFC volume in youth is only characteristic of more severe PTSD, although this would require further study with a sample of less-symptomatic youth.
Our findings suggest two areas of the vmPFC that may in part underlie the pathophysiology of pediatric PTSD. The first cluster, identified in the group contrast, was located in 
the anterior vmPFC (BA 10/11) in the medial frontopolar region. The frontopolar cortex is believed to have a role in allocating attentional resources to the processing of external $v s$ internal information (Burgess et al, 2007; Henseler et al, 2011). Consistent with its proposed functional role, our results revealed that anterior vmPFC volume was inversely associated with illness duration. In contrast, anterior vmPFC volume was not related to symptom severity. This suggests that reduced anterior vmPFC volume in pediatric PTSD may contribute more broadly to persistence of illness, rather than PTSD symptoms themselves, by a relative inability to direct attentional resources following a traumatic event. If this is the case, then anterior vmPFC volume may also influence response to psychotherapy-based treatment for pediatric PTSD. Interestingly, there were no agerelated differences in the anterior vmPFC, suggesting that reduced vmPFC volume may represent either a premorbid characteristic of pediatric PTSD or an acquired trait after trauma. Longitudinal imaging studies will ultimately be needed to examine these possibilities, by incorporating imaging before trauma exposure, as well as pre/posttreatment once PTSD has been identified.

The other vmPFC cluster associated with PTSD arose from a voxel-wise analysis of PTSD symptom cluster severity. Here, re-experiencing symptoms inversely correlated with GMV in the sgACC. As part of the vmPFC, the sgACC is thought to be a putative homolog of the rat infralimbic cortex (IL). The IL mediates recall of fear extinction by suppressing amygdala-based fear responses through activation of inhibitory intercalated neurons in the amygdala (Milad and Quirk, 2012). In humans, the vmPFC is normally recruited during fear extinction (Milad and Quirk, 2012; Phelps et al, 2004), whereas such recruitment is impaired in adult PTSD (Milad et al, 2009). A recent study from our group revealed that childhood maltreatment experiences are associated with lower functional connectivity between the sgACC and the amygdala and hippocampus. This weaker connectivity, in turn, mediated the development of adolescent-internalizing symptoms (Herringa et al, 2013). The current findings are consistent with these prior studies and suggest that lower sgACC volume is tied to greater re-experiencing symptoms, likely due to deficits in the ability to extinguish fear memories. Interestingly, there were no overall group or age-related differences in sgACC volume. This suggests that sgACC volume may not be a premorbid characteristic of pediatric PTSD, but that individual differences in sgACC GMV influence the expression of PTSD symptoms. As above, longitudinal imaging studies will be needed to explore these possibilities.

Our study also revealed age-related differences in hippocampus GMV in pediatric PTSD. Specifically, age positively predicted right anterior hippocampus GMV in healthy youth, but negatively predicted GMV in PTSD youth. One of the perplexing findings in prior studies of pediatric PTSD has been the lack of difference in hippocampal GMV in pediatric PTSD vs healthy youth, even though smaller hippocampal GMV has been repeatedly observed in adult PTSD (Pitman et al, 2012; Woon and Hedges, 2008). The current findings, although crosssectional, suggest an ongoing toxic process over development, whereby reduced hippocampal volume may not become fully apparent until adulthood. The mechanisms underlying abnormal hippocampal development are not entirely clear but may involve an interaction between childhood trauma exposure and genetic susceptibility. For example, childhood abuse exposure interacts with genetic polymorphisms of FKBP5, a glucocorticoid receptor regulatory protein, to differentially alter its methylation and lead to glucocorticoid receptor resistance (Klengel et al, 2013). Notably, FKBP5 methylation was also associated with volume of the right anterior hippocampus (Klengel et al, 2013), similar to the hippocampal area in the current study.

In the voxel-wise analysis of PTSD symptom cluster severity, we found that right anterior hippocampus volume, in addition to the sgACC, was inversely correlated with reexperiencing symptoms of PTSD. The hippocampus is capable of contextually gating fear responses via connections to both the amygdala and vmPFC (Maren et al, 2013). Along with the vmPFC, the hippocampus is recruited during the recall of fear extinction, for example (Kalisch et al, 2006; Milad et al, 2007), but shows impaired recruitment in adult PTSD (Milad et al, 2009). We have previously shown that childhood maltreatment experiences are associated with weaker hippocampus-sgACC connectivity, which in turn mediates the development of adolescent-internalizing symptoms (Herringa et al, 2013). The current findings suggest that this pathway may also have a role in the expression of symptoms in pediatric PTSD, presumably by impairing the contextual gating of fear memory. Further study will be needed to examine both the behavioral correlates of contextual fear regulation, as well as functional brain changes in this pathway in pediatric PTSD.

It is noteworthy that sgACC and hippocampal volumes were not related to total PTSD severity, but were associated with symptom clusters of PTSD. Our primary brain analyses point to re-experiencing symptoms being most directly tied to GMV in these areas, although post hoc analyses also suggest potential relationships with other symptom clusters. Here, sgACC and hippocampal volume were positively associated with avoidance symptoms, and marginally but differentially associated with hyperarousal symptoms. These findings suggest that the underlying neural substrates are unlikely to map on to the entire PTSD syndrome but may relate to specific symptom types, perhaps even in opposing ways within some neural circuits. Related to this, depressive symptoms could also be a contributing factor to vmPFC and hippocampal abnormalities in our sample, given the high rate of comorbid depression and previous work demonstrating reduced vmPFC and hippocampal GMV in major depressive disorder, for example (Kempton et al, 2011). Although our secondary analyses suggest that depressive symptoms do not directly relate to vmPFC and hippocampal abnormalities in our sample, future studies including a trauma-exposed group with depression (but no PTSD) would be warranted to explore common and differential neural pathways to these illnesses following trauma exposure.

The current study has revealed novel findings of reduced GMV and age-related differences in fear regulatory regions in pediatric PTSD. However, this study is not without limitations. First, the group comparisons are correlational by nature. Although we attempted to appropriately match 
subjects at the group level and covary for potentially confounding variables in secondary analyses, there could be other unidentified variables that differ between the groups and account for structural brain differences. Second, the current analyses are based on cross-sectional data, highlighting the need for future, longitudinal studies in both PTSD and healthy youth to supplement these findings and characterize brain developmental trajectories. Third, this is a moderate sample size, and our findings would merit replication in a larger sample of youth. Finally, our study did not contain a trauma-exposed, healthy comparison group. Although we attempted to explore the contribution of trauma exposure vs PTSD measures to brain findings in our secondary analyses, there could be other trauma-related measures that contribute to the current brain findings. Inclusion of healthy trauma-exposed youth in future work will be important for teasing apart these variables and exploring adaptive brain changes following trauma.

In summary, to our knowledge, this is the first report of morphological brain changes including age-related differences in pediatric PTSD using higher-resolution MRI and high-dimensional normalization along with VBM. Our findings suggest alterations in fear regulatory circuitry in pediatric PTSD including the vmPFC and hippocampus, and their link to re-experiencing symptoms of PTSD and illness duration. The negative relationship between age and hippocampal volume in pediatric PTSD may suggest an ongoing neurotoxic process over development. On the other hand, the lack of age-related differences in the vmPFC compared with healthy youth suggests that vmPFC volume may represent a premorbid or acquired trait that in turn influences illness course and symptom expression. Future studies employing longitudinal designs should aim to delineate brain developmental trajectories following pediatric trauma and PTSD, and determine whether successful treatments may normalize vmPFC and hippocampal abnormalities.

\section{FUNDING AND DISCLOSURE}

Funding for this study was provided by the American Academy of Child and Adolescent Psychiatry Junior Investigator Award $(\mathrm{RJH})$, NARSAD Young Investigator Grant (RJH), University of Wisconsin Institute for Clinical and Translational Research Translational Pilot Grant Award (NIH/NCATS UL1TR000427, RJH), National Institute of Mental Health Career Development Award (K08 MH100267, $\mathrm{RJH}$ ), and the University of Wisconsin School of Medicine and Public Health. Funding sources did not have any direct role in study design, analysis or interpretation of data, or preparation of the manuscript. The authors declare no conflict of interest.

\section{ACKNOWLEDGEMENTS}

We would like to thank Rachael Meline, Allison Blumenfeld, Ariel Sherman, and Erika Vassar-Olsen for their assistance in study recruitment and data collection, and Drs Richard Davidson and Ned Kalin for editorial comments on the manuscript.

\section{REFERENCES}

Apfel BA, Ross J, Hlavin J, Meyerhoff DJ, Metzler TJ, Marmar CR et al (2011). Hippocampal volume differences in gulf war veterans with current versus lifetime posttraumatic stress disorder symptoms. Biol Psychiatry 69: 541-548.

Birmaher B, Khetarpal S, Brent D, Cully M, Balach L, Kaufman J et al (1997). The Screen for Child Anxiety Related Emotional Disorders (SCARED): scale construction and psychometric characteristics. J Am Acad Child Adolesc Psychiatry 36: 545-553.

Bryant RA, Felmingham K, Whitford TJ, Kemp A, Hughes G, Peduto A et al (2008). Rostral anterior cingulate volume predicts treatment response to cognitive-behavioural therapy for posttraumatic stress disorder. J Psychiatry Neurosci 33: 142-146.

Burgess PW, Dumontheil I, Gilbert SJ (2007). The gateway hypothesis of rostral prefrontal cortex (area 10) function. Trends Cogn Sci 11: 290-298.

Carrion VG, Weems CF, Eliez S, Patwardhan A, Brown W, Ray RD et al (2001). Attenuation of frontal asymmetry in pediatric posttraumatic stress disorder. Biol Psychiatry 50: 943-951.

Carrion VG, Weems CF, Ray R, Reiss AL (2002). Toward an empirical definition of pediatric PTSD: the phenomenology of PTSD symptoms in youth. J Am Acad Child Adolesc Psychiatry 41: 166-173.

Carrion VG, Weems CF, Reiss AL (2007). Stress predicts brain changes in children: a pilot longitudinal study on youth stress, posttraumatic stress disorder, and the hippocampus. Pediatrics 119: $509-516$.

Carrion VG, Weems CF, Watson C, Eliez S, Menon V, Reiss AL (2009). Converging evidence for abnormalities of the prefrontal cortex and evaluation of midsagittal structures in pediatric posttraumatic stress disorder: an MRI study. Psychiatry Res 172: 226-234.

Cohen JA, Mannarino AP, Iyengar S (2011). Community treatment of posttraumatic stress disorder for children exposed to intimate partner violence: a randomized controlled trial. Arch Pediatr Adolesc Med 165: 16-21.

Costello EJ, Angold A (1988). Scales to assess child and adolescent depression: checklists, screens, and nets. J Am Acad Child Adolesc Psychiatry 27: 726-737.

Cox RW (1996). AFNI: software for analysis and visualization of functional magnetic resonance neuroimages. Comput Biomed Res 29: 162-173.

De Bellis MD, Keshavan MS (2003). Sex differences in brain maturation in maltreatment-related pediatric posttraumatic stress disorder. Neurosci Biobehav Rev 27: 103-117.

De Bellis MD, Keshavan MS, Clark DB, Casey BJ, Giedd JN, Boring AM et al (1999). A.E. Bennett Research Award. Developmental traumatology. Part II: Brain development. Biol Psychiatry 45: 1271-1284.

De Bellis MD, Keshavan MS, Shifflett H, Iyengar S, Beers SR, Hall J et al (2002). Brain structures in pediatric maltreatment-related posttraumatic stress disorder: a sociodemographically matched study. Biol Psychiatry 52: 1066-1078.

Dickie EW, Brunet A, Akerib V, Armony JL (2013). Anterior cingulate cortical thickness is a stable predictor of recovery from post-traumatic stress disorder. Psychol Med 43: 645-653.

Famularo R, Fenton T, Kinscherff R, Augustyn M (1996). Psychiatric comorbidity in childhood post traumatic stress disorder. Child Abuse Negl 20: 953-961.

Gilbertson MW, Shenton ME, Ciszewski A, Kasai K, Lasko NB, Orr SP et al (2002). Smaller hippocampal volume predicts pathologic vulnerability to psychological trauma. Nat Neurosci 5: 1242-1247.

Henseler I, Krüger S, Dechent P, Gruber O (2011). A gateway system in rostral PFC? Evidence from biasing attention to perceptual information and internal representations. Neuroimage 56: 1666-1676.

Herringa RJ, Birn RM, Ruttle PL, Burghy CA, Stodola DE, Davidson RJ et al (2013). Childhood maltreatment is associated 
with altered fear circuitry and increased internalizing symptoms by late adolescence. Proc Natl Acad Sci USA 110: 19119-19124.

Herringa R, Phillips M, Almeida J, Insana S, Germain A (2012). Post-traumatic stress symptoms correlate with smaller subgenual cingulate, caudate, and insula volumes in unmedicated combat veterans. Psychiatry Res 203: 139-145.

Kalisch R, Korenfeld E, Stephan KE, Weiskopf N, Seymour B, Dolan RJ (2006). Context-dependent human extinction memory is mediated by a ventromedial prefrontal and hippocampal network. J Neurosci 26: 9503-9511.

Karl A, Schaefer M, Malta LS, Dorfel D, Rohleder N, Werner A (2006). A meta-analysis of structural brain abnormalities in PTSD. Neurosci Biobehav Rev 30: 1004-1031.

Kasai K, Yamasue H, Gilbertson MW, Shenton ME, Rauch SL, Pitman RK (2008). Evidence for acquired pregenual anterior cingulate gray matter loss from a twin study of combat-related posttraumatic stress disorder. Biol Psychiatry 63: 550-556.

Kaufman J, Birmaher B, Brent D, Rao U, Flynn C, Moreci P et al (1997). Schedule for Affective Disorders and Schizophrenia for School-Age Children-Present and Lifetime Version (K-SADSPL): initial reliability and validity data. J Am Acad Child Adolesc Psychiatry 36: 980-988.

Kempton MJ, Salvador Z, Munafò MR, Geddes JR, Simmons A, Frangou $S$ et al (2011). Structural neuroimaging studies in major depressive disorder. Meta-analysis and comparison with bipolar disorder. Arch Gen Psychiatry 68: 675-690.

Klengel T, Mehta D, Anacker C, Rex-Haffner M, Pruessner JC, Pariante CM et al (2013). Allele-specific FKBP5 DNA demethylation mediates gene-childhood trauma interactions. Nat Neurosci 16: $33-41$

Kühn S, Gallinat J (2013). Gray matter correlates of posttraumatic stress disorder: a quantitative meta-analysis. Biol Psychiatry 73: 70-74.

Levy-Gigi E, Szabó C, Kelemen O, Kéri S (2013). Association among clinical response, hippocampal volume, and FKBP5 gene expression in individuals with posttraumatic stress disorder receiving cognitive behavioral therapy. Biol Psychiatry 74: 793-800.

Maren S, Phan KL, Liberzon I (2013). The contextual brain: implications for fear conditioning, extinction and psychopathology. Nat Rev Neurosci 14: 417-428.

Meng Y, Qiu C, Zhu H, Lama S, Lui S, Gong Q et al (2014). Anatomical deficits in adult posttraumatic stress disorder: a meta-analysis of voxel-based morphometry studies. Behav Brain Res 270C: 307-315.

Merikangas KR, He J-P, Burstein M, Swanson SA, Avenevoli S, Cui L et al (2010). Lifetime prevalence of mental disorders in U.S. adolescents: results from the National Comorbidity Survey Replication-Adolescent Supplement (NCS-A). J Am Acad Child Adolesc Psychiatry 49: 980-989.

Milad MR, Pitman RK, Ellis CB, Gold AL, Shin LM, Lasko NB et al (2009). Neurobiological basis of failure to recall extinction memory in posttraumatic stress disorder. Biol Psychiatry 66: 1075-1082.

Milad MR, Quirk GJ (2012). Fear extinction as a model for translational neuroscience: ten years of progress. Annu Rev Psychol 63: 129-151.

Milad MR, Wright CI, Orr SP, Pitman RK, Quirk GJ, Rauch SL (2007). Recall of fear extinction in humans activates the ventromedial prefrontal cortex and hippocampus in concert. Biol Psychiatry 62: 446-454.
Morey RA, Gold AL, LaBar KS, Beall SK, Brown VM, Haswell CC et al (2012). Amygdala volume changes in posttraumatic stress disorder in a large case-controlled veterans group. Arch Gen Psychiatry 69: 1169-1178.

Morris NM, Udry JR (1980). Validation of a self-administered instrument to assess stage of adolescent development. $J$ Youth Adolescence 9: 271-280.

Nader KO, Newman E, Weathers FW, Kaloupek DG, Kriegler JA, Blake DD et al (1998). Clinician-Administered PTSD Scale for Children and Adolescents for DSM-IV. National Center for PTSD: White River Junction, VT.

Norrholm SD, Jovanovic T, Olin IW, Sands LA, Karapanou I, Bradley B et al (2011). Fear extinction in traumatized civilians with posttraumatic stress disorder: relation to symptom severity. Biol Psychiatry 69: 556-563.

Phelps EA, Delgado MR, Nearing KI, LeDoux JE (2004). Extinction learning in humans: role of the amygdala and vmPFC. Neuron 43: 897-905.

Pitman RK, Rasmusson AM, Koenen KC, Shin LM, Orr SP, Gilbertson MW et al (2012). Biological studies of post-traumatic stress disorder. Nat Rev Neurosci 13: 769-787.

Richert KA, Carrion VG, Karchemskiy A, Reiss AL (2006). Regional differences of the prefrontal cortex in pediatric PTSD: an MRI study. Depress Anxiety 23: 17-25.

Sekiguchi A, Sugiura M, Taki Y, Kotozaki Y, Nouchi R, Takeuchi H et al (2013). Brain structural changes as vulnerability factors and acquired signs of post-earthquake stress. Mol Psychiatry 18: 618-623.

Steinberg AM, Brymer MJ, Decker KB, Pynoos RS (2004). The University of California at Los Angeles Post-traumatic Stress Disorder Reaction Index. Curr Psychiatry Rep 6: 96-100.

Uematsu A, Matsui M, Tanaka C, Takahashi T, Noguchi K, Suzuki M et al (2012). Developmental trajectories of amygdala and hippocampus from infancy to early adulthood in healthy individuals. PLoS One 7: e46970.

Vermetten E, Vythilingam M, Southwick SM, Charney DS, Bremner JD (2003). Long-term treatment with paroxetine increases verbal declarative memory and hippocampal volume in posttraumatic stress disorder. Biol Psychiatry 54: 693-702.

Warshaw MG, Fierman E, Pratt L, Hunt M, Yonkers KA, Massion AO et al (1993). Quality of life and dissociation in anxiety disorder patients with histories of trauma or PTSD. Am J Psychiatry 150: $1512-1516$.

Weathers FW, Keane TM, Davidson JR (2001). Clinician-administered PTSD scale: a review of the first ten years of research. Depress Anxiety 13: 132-156.

Wechsler D (2011). Wechsler Abbreviated Scale of IntelligenceSecond Edition Manual. Pearson: Bloomington, MN.

Woon FL, Hedges DW (2008). Hippocampal and amygdala volumes in children and adults with childhood maltreatment-related posttraumatic stress disorder: a meta-analysis. Hippocampus 18: 729-736.

Woon FL, Hedges DW (2009). Amygdala volume in adults with posttraumatic stress disorder: a meta-analysis. J Neuropsychiatry Clin Neurosci 21: 5-12.

Woon FL, Sood S, Hedges DW (2010). Hippocampal volume deficits associated with exposure to psychological trauma and posttraumatic stress disorder in adults: a meta-analysis. Prog Neuropsychopharmacol Biol Psychiatry 34: 1181-1188.

Supplementary Information accompanies the paper on the Neuropsychopharmacology website (http://www.nature.com/npp) 